DOI: $10 / 15593 / 2224-9877 / 2016.2 .03$

УДК 620.179 .18

\author{
В.Ф. Новиков, Д.Ф. Нерадовский, Р.А. Соколов \\ Тюменский индустриальный университет, Тюмень, Россия \\ ИСПОЛЬЗОВАНИЕ КВАЗИСТАТИЧЕСКИХ ПЕТЕЛЬ \\ МАГНИТНОГО ГИСТЕРЕЗИСА ДЛЯ КОНТРОЛЯ \\ СТРУКТУРЫ СТАЛИ
}

\begin{abstract}
В процессе эксплуатации изделия из стали подвергаются различным механическим и термическим воздействиям, что вызывает рост усталостных изменений, вследствие чего происходит преждевременное старение материала, зачастую приводящее к техногенным катастрофам. В связи с этим важно контролировать свойства стали и изделий из стали. Методика, предлагаемая в качестве дополнительного средства контроля, основана на явлении магнитного гистерезиса и была опробована на стали марки 34ХНЗМ. Данная сталь применяется для изготовления деталей, испытывающих в процессе эксплуатации высокие давления и температуры до $500{ }^{\circ} \mathrm{C}$.

Предлагается использовать петли магнитного гистерезиса ферромагнетиков, полученных путем квазистатического перемагничивания. В этом случае ток намагничивания и размагничивания, а значит, и напряженность магнитного поля описываются пилообразной функцией времени. Тогда становится возможным представить петлю гистерезиса в виде однозначной функции напряженности поля, пропорциональной времени. Для полученных функций выполнено дискретное преобразование Фурье.

В данной работе исследуются корреляционные зависимости Фурье-спектра петель гистерезиса стали от структуры и механических свойств стали. К структурным изменениям в стали при ее термической обработке можно отнести, например, снятие напряжений, выделение карбидов и их изменение. Указанные превращения в стали существенно сказываются на форме петли, а так как гармонический Фурье-спектр чувствителен к изменению формы сигнала, то метод Фурье-анализа может служить дополнительным средством неразрушающего контроля структурных изменений в сталях, вместе с измерением коэрцитивной силы, твердости и некоторых других параметров.
\end{abstract}

Ключевые слова: гармонический анализ, магнитная структуроскопия, магнитный гистерезис, коэрцитивная сила, твердость, аустенит, мартенсит, амплитуда гармоник, температура отпуска, твердый раствор.

\author{
V.F. Novikov, D.F. Neradovskii, R.A. Sokolov \\ Tyumen Industrial University, Tyumen, Russian Federation
}

\title{
THE USING OF QUASI-STATIC MAGNETIC HYSTERESIS LOOPS TO CONTROL STEEL STRUCTURES
}

During the operation, steel products are under various mechanical and thermal impacts, that can cause the growth of fatigue changes, and as a result, premature aging of the material can happen. It leads to technosphere disasters. That's why, it is important to control the properties of steel and steel 
products. The method proposed as an additional means of control is based on the phenomenon of magnetic hysteresis and was tested on steel $34 \mathrm{HN} 3 \mathrm{M}$. This steel is used for manufacturing of different components operating at temperatures up to $500{ }^{\circ} \mathrm{C}$ and high pressure.

It is suggested to use a magnetic hysteresis loop of ferromagnetic materials, obtained by quasistatic magnetization. In this case, the current magnetization and demagnetization and hence the magnetic field, is described sawtooth function of time. Then it becomes possible to provide a hysteresis loop in the form of a single-valued function of the field strength that is proportional to the time period. Discrete Fourier transformation is applied for the obtain the given functions.

In this paper we study the correlations of the Fourier spectrum of the hysteresis loops of steel, dependent on a change of its structure and mechanical properties. The structural changes in the steel during its thermal processing may include, for example: stress relief, precipitation of carbides and their changing. The aforementioned changes in steel greatly affects the shape of the loop, out as a harmonic Fourier spectrum is sensitive to the waveform change, the method of Fourier analysis may provide an additional means of nondestructive inspection of structural changes in the steels, together with the measurement of coercive force, hardness and other parameters.

Keywords: harmonic analysis, magnetic structurescopy, magnetic hysteresis, coercive force, hardness, austenite, martensite, amplitude of the harmonics, tempering temperature, solid solution.

Свойства магнитных материалов зависят от их химического состава, способа изготовления и термической обработки.

Магнитные свойства материала могут изменяться в процессе изготовления, механической или термической обработки, когда происходят изменения структуры материала. Следовательно, для решения вопроса о методике получения магнитных материалов с заданными свойствами важную роль играет изучение процессов, происходящих в строении магнитного материала.

Механические и термические воздействия на сталь на этапах производства и эксплуатации изделий часто вызывают рост усталостных изменений, что приводит к преждевременному старению материала и, как следствие, различного рода техногенным катастрофам.

На производстве причинами, приводящими к внутренним напряжениям, возникающим при пластической деформации, являются протяжка, прокатка или волочение. При этом происходит уменьшение магнитной проницаемости материала [1]. После такой обработки материал находится в неоднородно-напряженном состоянии, напоминающем напряженное состояние после фазового превращения. Напряжения в материале почти всегда можно снять посредством отжига для повышения проницаемости материалов, пластически деформированных при изготовлении.

Также для магнитных параметров важно влияние примесей. На рис. 1 представлены кривые намагничивания сталей с различным содержанием углерода: нижняя кривая получена на малоуглеродистой (мягкой) стали, содержащей 0,2 \% C; средняя - на электротехнической 
стали, содержащей около $0,02 \% \mathrm{C}$ и отожженной примерно при $900{ }^{\circ} \mathrm{C}$. Если данную сталь отжигать в течение нескольких часов при $1400{ }^{\circ} \mathrm{C}$, то содержание углерода уменьшается в ней до $0,001 \%$, другие примеси также удаляются. Очищенный таким способом материал дает верхнюю кривую. На рис. 1 использованы следующие обозначения: $B$ - индукция внутреннего магнитного поля, измеренная в гауссах (1 Гс $=10^{-4}$ Тл); $H$ - напряженность внешнего намагничивающего по-

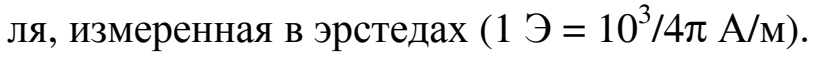

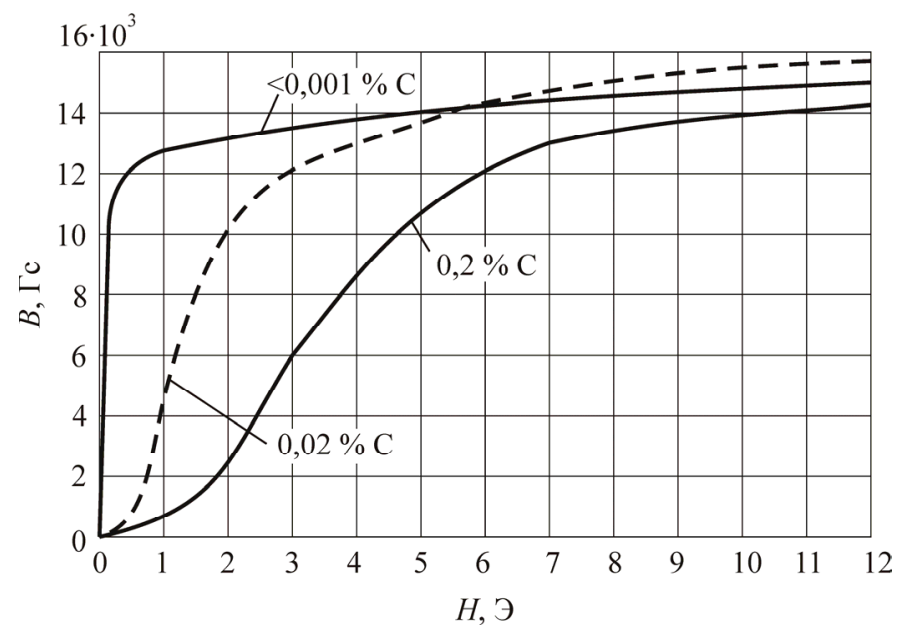

Рис. 1. Влияние примесей на магнитные свойства железа (отжиг в водороде при температуре $1400{ }^{\circ} \mathrm{C}$ уменьшает содержание углерода с 0,02 до $0,001 \%$ и менее) [1]

Контроль свойств стали является неотъемлемым процессом на производстве. Для контроля структуры и качества магнитных сталей используются магнитные методы нарушающего контроля, составляющие основу магнитной структуроскопии.

При магнитной структуроскопии для контроля состояния материала снимаются петли магнитного гистерезиса, состоящие из большого числа экспериментальных точек. Но только некоторые из этих точек используются для целей неразрушающего контроля: коэрцитивная сила, магнитная проницаемость, релаксационная коэрцитивная сила, дифференциальная магнитная проницаемость и др.

Приведенные выше магнитные параметры ферромагнетика, а также механические и структурные свойства связаны корреляцион- 
ной зависимостью [2-4], которая устанавливается экспериментально. Часто коэффициенты корреляции для этих зависимостей не очень велики.

В структуроскопии также хорошо известны и апробированы методы, основанные на анализе ЭДС индукции в измерительной катушке с помощью цифрового анализатора спектра или дискретного преобразования Фурье [5]. Полученные гармоники Фурье-спектра ЭДС связываются с изменением структурных свойств материала. В этом случае фактически используются все точки петли магнитного гистерезиса.

Однако при таком виде анализа существуют факторы, снижающие достоверность результатов, а именно наличие вихревых токов, которые ограничивают глубину промагничивания и искажают амплитуду и фазу выходного сигнала.

Целью работы является исследование возможности использования преимуществ гармонического анализа петель магнитного гистерезиса, полученных методом квазистатического перемагничивания.

Квазистатические петли гистерезиса ферромагнетиков можно снимать с помощью современных магнитометров (например, модель КРМ-Ц-МА). В такого рода приборах (как в предлагаемой работе) ток намагничивания и размагничивания, а следовательно, и напряженность поля описываются некоторой пилообразной функцией. Это позволяет сделать замену $t=H / k$. Симметрично отобразим нисходящую (верхнюю) ветвь петли относительно вертикальной прямой, проходящей через точку $H_{\max }$ (рис. 2). Таким образом, получается представление петли гистерезиса в виде функции $f(H)=f(k, t)$ для которой может быть выполнено дискретное преобразование Фурье $[6,7]$.

Рассчитаны амплитуды 1-й, 3-й, 5-й, 7-й гармоник петель гистерезиса стали 34ХН3М в зависимости от температуры отпуска (рис. 3). На этом рисунке буквой $A$ обозначены безразмерные амплитуды гармоник Фурье-спектра. 

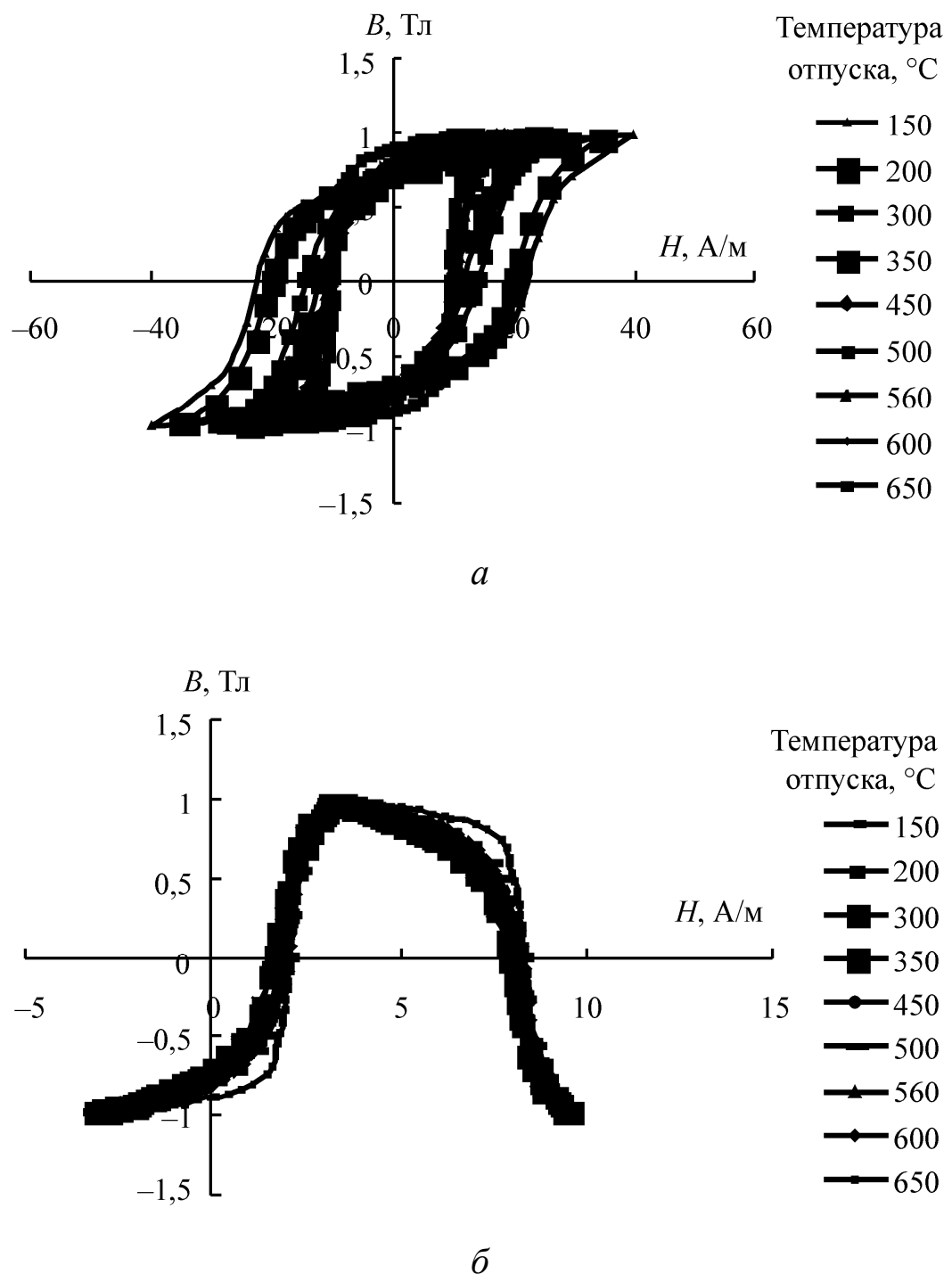

Рис. 2. Петли гистерезиса стали $34 \mathrm{XH3М,} \mathrm{отпущенной} \mathrm{при} \mathrm{различных}$ температурах: $a$ - в исходном виде; $\sigma$ - развернутые петли, приведенные к периоду $2 \pi$ 


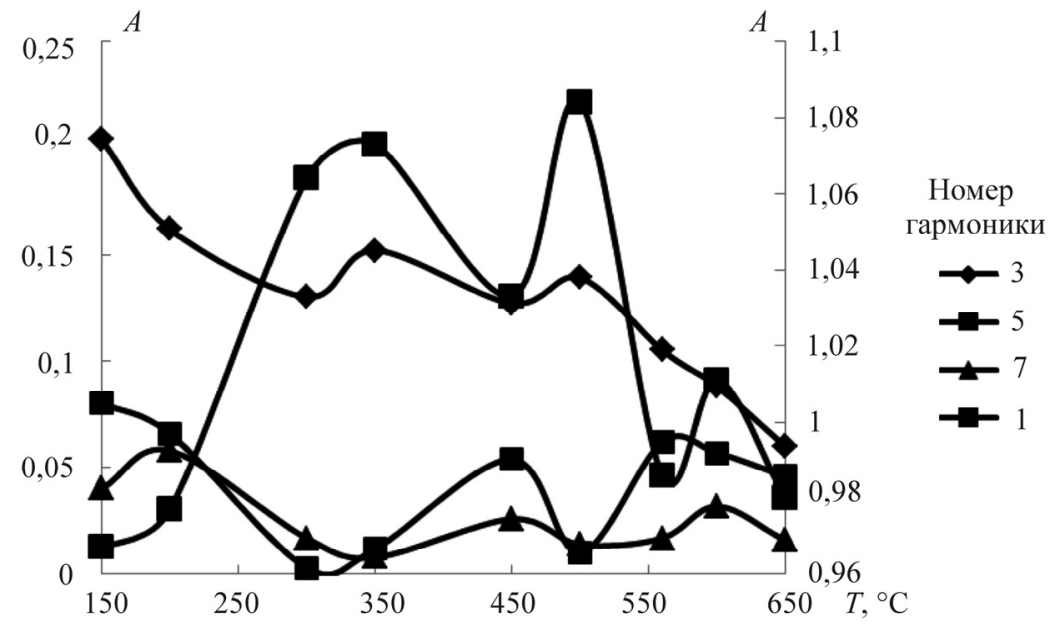

Рис. 3. Зависимости амплитуд нечетных гармоник Фурье-спектра петель гистерезиса для стали 34ХН3М от температуры отпуска

В работах $[2,8]$ показано, что в диапазоне температур отпуска от 150 до $250{ }^{\circ} \mathrm{C}$ для стали $34 \mathrm{XH} 3 \mathrm{M}$ наблюдается изменение намагниченности насыщения, обусловленное процессом распада мартенсита, интенсивно протекающим при температуре около $200{ }^{\circ} \mathrm{C}$, продолжающимся при более высоких температурах, заходящим в область температур $300-325{ }^{\circ} \mathrm{C}$, и одновременным процессом распада остаточного аустенита и снятием закалочных напряжений.

Такой отпуск сопровождается резким изменением структуры стали и, соответственно, изменением физических свойств [9], например коэрцитивной силы и твердости, зависимости для которых приведены на рис. 4.

Из приведенного графика для амплитуд 3-й, 5-й, 7-й гармоник можно заметить, что эти амплитуды существенно убывают в данном диапазоне температур. Сравнивая зависимости амплитуд гармоник и величины коэрцитивной силы и твердости от температуры отпуска, можно заметить корреляционную зависимость между их величинами.

При дальнейшем повышении температуры отпуска в диапазоне $250-450{ }^{\circ} \mathrm{C}$ происходит распад мартенсита на цементит и феррит, сопровождающийся уменьшением закалочных напряжений, также происходит рост карбидных частиц, форма их начинает приближаться к равноосной [10]. Искажения решетки твердого раствора снижаются. Закономерная ориентировка решеток $\alpha$-фазы и цементита сохраняется 
до температуры $650-680{ }^{\circ} \mathrm{C}[11,12]$. В этом диапазоне происходит небольшой рост амплитуд 3-й, 5-й, 7-й гармоник, а также замедление падения величины коэрцитивной силы. Для амплитуды 1-й гармоники в данном диапазоне температур наблюдается рост и падение.

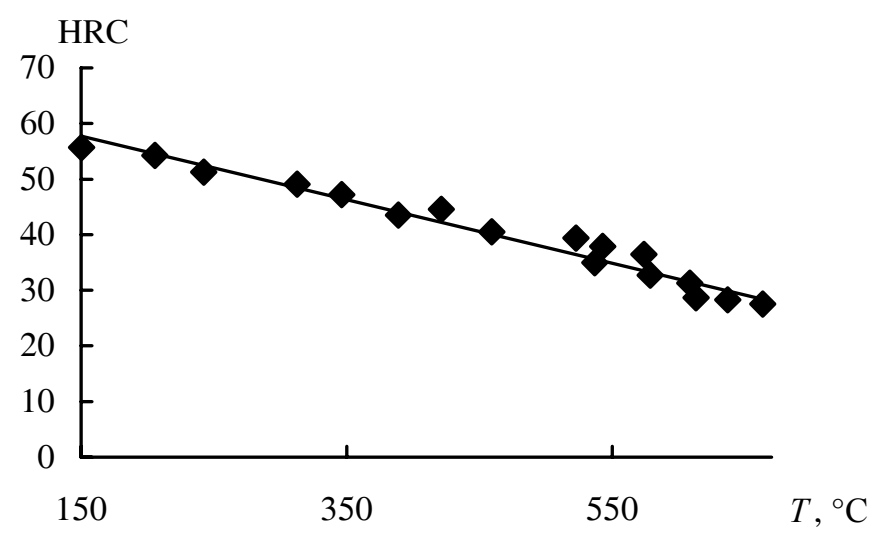

$a$

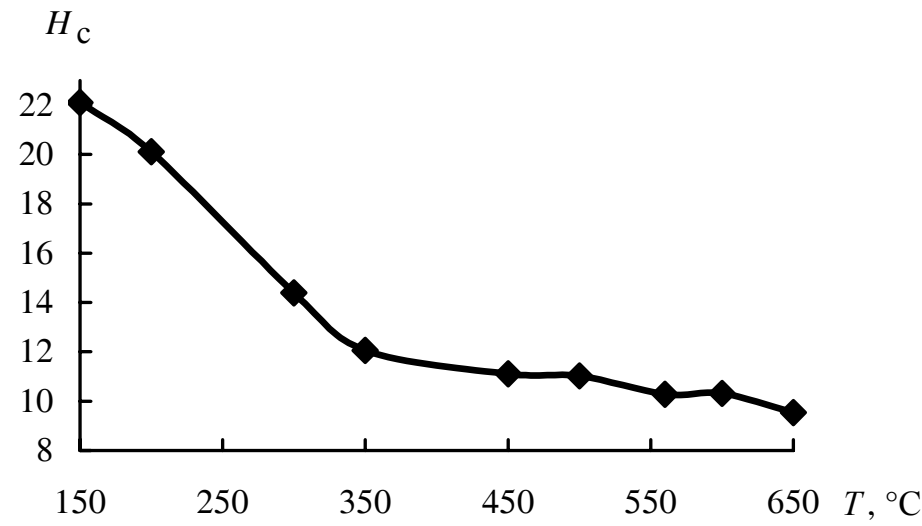

$\sigma$

Рис. 4. Величина твердости (a) и коэрцитивной силы (б) для стали $34 \mathrm{XH} 3 \mathrm{M}$, отпущенной при различных температурах

В интервале температур $450-650{ }^{\circ} \mathrm{C}$ происходит рекристаллизация матрицы, легирование цементита карбидообразующими химическими элементами, очищение решетки $\alpha-\mathrm{Fe}$ от углерода и коагуляция карбидных частиц, т.е. растворение мелких карбидных частиц и рост более крупных, также происходит снижение пластических свойств 
и ударной вязкости, что объясняется дроблением блоков $\alpha$-фазы. Эти процессы взаимосвязаны и ведут к росту среднего размера частиц [13]. При температурах 550-600 ${ }^{\circ} \mathrm{C}$ имеет место растворение карбидов из-за повышения предела растворимости углерода в $\alpha-\mathrm{Fe}$, происходит снятие напряжений, обусловленное завершением процесса измельчения блоков, а следовательно, и устранение искажений решетки, вызванных их связанностью [14]. На графиках этому соответствует падение амплитуды нечетных гармоник, а также дальнейшее падение величины твердости и коэрцитивной силы. Кроме того, можно заметить корреляционную зависимость между амплитудой 5-й гармоники и величиной коэрцитивной силы в этом интервале температур, которая проявляется в возрастании значений этих величин при температуре 500 и $600{ }^{\circ} \mathrm{C}$.

В интервале температур $350-550{ }^{\circ} \mathrm{C}$ могут происходить коагуляция и сфероизация карбидных частиц, связанные с повышением температуры или продолжительности отпуска $[14,15]$. В этой области температур наблюдается рост величины амплитуды 7-й гармоники. Пик дисперсионных напряжений, обусловленный обособлением кристаллов карбидов от твердого раствора, приходится на диапазон 400$500{ }^{\circ} \mathrm{C}$. В данной области температур амплитуда 1-й гармоники возрастает.

Таким образом, можно говорить о том, что представленные результаты отражают чувствительность гармонических составляющих к структурным изменениям в стали и соответствующим им изменениям твердости и коэрцитивной силы, а именно снятие напряжений, выделение карбидов, их изменения в результате увеличения температуры отпуска, что может служить дополнительным средством неразрушающего контроля структурных изменений в сталях.

\section{Список литературы}

1. Михеев М.Н., Горкунов Э.С. Магнитные методы структурного анализа и неразрушающего контроля. - М.: Наука, 1993. - С. 50-75.

2. Бида Г.В., Горкунов Э.С., Шевнин В.М. Магнитный контроль механических свойств проката. - Екатеринбург: Изд-во УрО РАН, 2002. $-252 \mathrm{c}$.

3. Пономарев Ю.Ф. Гармонический анализ намагниченности ферромагнетиков, перемагничиваемых переменным полем, с учетом 
магнитного гистерезиса. IV. Применение к неразрушающему контролю // Дефектоскопия. - 1986. - № 7. - С. 67-78.

4. Белашов В.Ю., Чернова Н.М. Эффективные алгоритмы и программы вычислительной математики / Сев.-Вост. комплекс. науч.исслед. ин-т им. Н.А. Шило ДВО РАН. - Владивосток, 1997. - 160 с.

5. Бозорт Р. Ферромагнетизм. - М.: Изд-во иностр. лит-ры, 1956. - C. 5-15.

6. Бахвалов Н.С., Жидков Н.П., Кобельков Г.М. Численные методы. - М.: Бином. Лаборатория знаний, 2003. - 484 с.

7. Горкунов Э.С., Михеев М.Н., Дунаев Ф.Н. Магнитные и электрические свойства сталей 18ХНВА, 34ХН3М и У9А в зависимости от режима термообработки // Дефектоскопия. - 1975. - № 3. - С. 119-126.

8. Бида Г.В., Ничипурук А.П. Магнитные свойства термообработанных сталей. - Екатеринбург: Изд-во УрО РАН, 2005. - С. 79-84.

9. Структура и физико-механические свойства сталей / М.Н. Михеев, В.М. Морозова, Н.И. Носкова [и др.]; Ин-т физики металлов Урал. науч. центра АН СССР. - Свердловск, 1981. - 31 с.

10. Могутнов Б.М., Томилин И.А., Шварцман Л.А. Термодинамика сплавов железа. - М.: Металлургия, 1984. - 208 с.

11. Михеев М.Н., Горкунов Э.С. Магнитные методы неразрушающего контроля структурного состояния и прочностных характеристик термически обработанных изделий // Дефектоскопия. - 1985. № 3. - С. 3-21.

12. Кузнецов И.А., Михеев М.Н. Магнитные, электрические и механические свойства высокохромистых сталей после различных термических обработок // ФММ. - 1959. - Т. 7, № 4. - С. 514-526.

13. Курдюмов В.Г., Утевский Л.М., Энтин Р.И. Превращения в железе и стали. - М.: Наука, 1977. - 238 с.

14. Белоусов М.В., Черепин В.Т., Васильев М.А. Превращения при отпуске стали. - М.: Металлургия, 1973. - 272 с.

15. Бида Г.В., Сажина Е.Ю., Царьков Т.П. Магнитные свойства и возможность неразрушающего контроля закаленных и отпущенных высокохромистых сталей // Дефектоскопия. - 1996. - № 8. - С. 21-29. 


\section{References}

1. Mikheev M.N., Gorkunov E.S. Magnitnye metody strukturnogo analiza i nerazrushaiushchego kontrolia [Magnetic methods of the structural analysis and nondestructive control]. Moscow: Nauka, 1993, pp. 50-75.

2. Bida G.V., Gorkunov E.S., Shevnin V.M. Magnitnyi kontrol' mekhanicheskikh svoistv prokata [Magnetic control of mechanical properties of hire]. Ekaterinburg: Ural'skoe otdelenie Rossiiskoi akademii nauk, 2002. $252 \mathrm{p}$.

3. Ponomarev Iu.F. Garmonicheskii analiz namagnichennosti ferromagnetikov, peremagnichivaemykh peremennym polem, s uchetom magnitnogo gisterezisa. IV. Primenenie $\mathrm{k}$ nerazrushaiushchemu kontroliu [The harmonious analysis of magnetization of the ferkromagnetik remagnetized by a variation field taking into account a magnetic hysteresis. IV. Application to nondestructive control]. Defektoskopiia, 1986, no. 7, pp. 67-78.

4. Belashov V.Iu., Chernova N.M. Effektivnye algoritmy i programmy vychislitel'noi matematiki [Effective algorithms and programs of calculus mathematics]. Vladivostok: Severo-Vostochnyi kompleksnyi nauchnoissledovatel'skii institut imeni N.A. Shilo Dal'nevostochnogo otdeleniia Rossiiskoi akademii nauk, 1997. 160 p.

5. Bozort R. Ferromagnetizm [Ferromagnetics]. Moscow: Izdatel'stvo inostrannoi literatury, 1956, pp. 5-15.

6. Bakhvalov N.S., Zhidkov N.P., Kobel'kov G.M. Chislennye metody [Numerical methods]. Moscow: Binom. Laboratoriia znanii, 2003. 484 p.

7. Gorkunov E.S., Mikheev M.N., Dunaev F.N. Magnitnye i elektricheskie svoistva stalei $18 \mathrm{KhNVA}, 34 \mathrm{KhN} 3 \mathrm{M}$ i U9A v zavisimosti ot rezhima termoobrabotki [Magnetic and electric properties staly 18HNVA, 34HN3M and U9A depending on the heat treatment mode]. Defektoskopiia, 1975, no. 3, pp. 119-126.

8. Bida G.V., Nichipuruk A.P. Magnitnye svoistva termoobrabotannykh stalei [Magnetic properties thermoprocessed by staly]. Ekaterinburg: Ural'skoe otdelenie Rossiiskoi akademii nauk, 2005, pp. 79-84.

9. Mikheev M.N., Morozova V.M., Noskova N.I. [et al.]. Struktura i fiziko-mekhanicheskie svoistva stalei [Structure and physicomechanical properties of staly]. Sverdlovsk: Institut fiziki metallov Ural'skogo nauchnogo tsentra Akademii nauk SSSR, 1981. 31 p. 
10. Mogutnov B.M., Tomilin I.A., Shvartsman L.A. Termodinamika splavov zheleza [Thermodynamics of alloys of iron]. Moscow: Metallurgiia, 1984. 208 p.

11. Mikheev M.N., Gorkunov E.S. Magnitnye metody nerazrushaiushchego kontrolia strukturnogo sostoianiia i prochnostnykh kharakteristik termicheski obrabotannykh izdelii [Magnetic methods of nondestructive control of a structural state and strength characteristics of thermally processed products]. Defektoskopiia, 1985, no. 3, pp. 3-21.

12. Kuznetsov I.A., Mikheev M.N. Magnitnye, elektricheskie i mekhanicheskie svoistva vysokokhromistykh stalei posle razlichnykh termicheskikh obrabotok [Magnetic, electric and mechanical properties highchromium staly after various heat treatments]. Fizika metallov $i$ metallovedenie, 1959, vol. 7, no. 4, pp. 514-526.

13. Kurdiumov V.G., Utevskii L.M., Entin R.I. Prevrashcheniia v zheleze i stali [Transformations in iron and steel]. Moscow: Nauka, 1977. $238 \mathrm{p}$.

14. Belousov M.V., Cherepin V.T., Vasil'ev M.A. Prevrashcheniia pri otpuske stali [Transformations at holiday became]. Moscow: Metallurgiia, 1973. $272 \mathrm{p}$.

15. Bida G.V., Sazhina E.Iu., Tsar'kov T.P. Magnitnye svoistva i vozmozhnost' nerazrushaiushchego kontrolia zakalennykh i otpushchennykh vysokokhromistykh stalei [Magnetic properties and a possibility of nondestructive control tempered and released high-chromium by staly]. Defektoskopiia, 1996, no. 8, pp. 21-29.

Получено 16.03.2016

\section{Об авторах}

Новиков Виталий Федорович (Тюмень, Россия) - доктор физико-математических наук, профессор, заведующий кафедрой «Физика, методы контроля и диагностики» Тюменского индустриального университета; e-mail: Novik1937@mail.ru.

Нерадовский Денис Федорович (Тюмень, Россия) - старший преподаватель кафедры «Физика, методы контроля и диагностики» Тюменского индустриального университета; e-mail: denner81@mail.ru.

Соколов Роман Александрович (Тюмень, Россия) - студент, Тюменский индустриальный университет; e-mail: falcon.rs@ mail.ru. 


\begin{abstract}
About the authors
Vitalii F. Novikov (Tyumen, Russian Federation) - Doctor of Physical and Mathematical Sciences, Professor, Head of Department of Physics, Monitoring and Diagnostics, Tyumen Industrial University; e-mail: Novik1937@mail.ru.

Denis F. Neradovskii (Tyumen, Russian Federation) - Senior Lecturer, Department of Physics, Monitoring and Diagnostics, Tyumen Industrial University; e-mail: denner81@ mail.ru.

Roman A. Sokolov (Tyumen, Russian Federation) - Student, Tyumen Industrial University; e-mail: falcon.rs@mail.ru.
\end{abstract}

